\title{
NGC 3227 - An Interacting Barred Spiral with an Active Nucleus
}

\author{
C. G. Mundell, A. Pedlar and D. L. Shone
}

University of Manchester, Nuffield Radio Astronomy Laboratories, Jodrell Bank, Macclesfield, Cheshire SK11 9DL, U. K.

D. J. Axon

STScI, 3700 San Martin Drive, Baltimore, MD 21218

J. Meaburn

University of Manchester, Department of Astronomy, Manchester M13 $g P L, U . K$.

S. W. Unger

Royal Greenwich Observatory, Madingly Rd., Cambridge CB3 OEZ, U. $K$.

\section{Introduction}

Recently, bars have become a favored mechanism for transporting material at larger radii towards the nuclei of active galaxies, with the possibility of inflowing gas (on kiloparsec scales) accumulating in circumnuclear rings, bar-like features or structures reminiscent of spiral arms. In addition, it seems clear that interactions play some role in the triggering and fuelling of nuclear activity, as well as in the formation and evolution of galactic bars. NGC 3227 is a barred Seyfert galaxy which appears to be interacting with the nearby elliptical galaxy NGC 3226 . The NGC 3227 system thus appears to be an excellent "laboratory case" in which to study the relationship between the bar phenomenon, and an active nucleus in an interacting system.

\section{Observations \& Discussion}

As part of a detailed optical and radio study (Mundell et al. 1995), we have used the VLA (C \& D-configurations) to image the neutral hydrogen emission from the NGC $3226 / 7$ system, with angular resolutions ranging from $12^{\prime \prime}$ to $60^{\prime \prime}$. The low-resolution images (Figure 1) show plumes of HI extending to $\sim 70$ $\mathrm{kpc}$ north and $\sim 31 \mathrm{kpc}$ south of the galaxy which may be a consequence of interaction. At higher resolution, the galactic disk and bar are resolved, and we see approximate solid-body rotation and an anomalous-velocity cloud ( $\sim 150 \mathrm{~km}$ $\mathrm{s}^{-1}$ above the systemic velocity of NGC 3227) situated northwest of the disk at the base of the northern plume. The cloud shows some evidence for rotation, and we suggest that this might be a gas-rich dwarf galaxy which may either be 


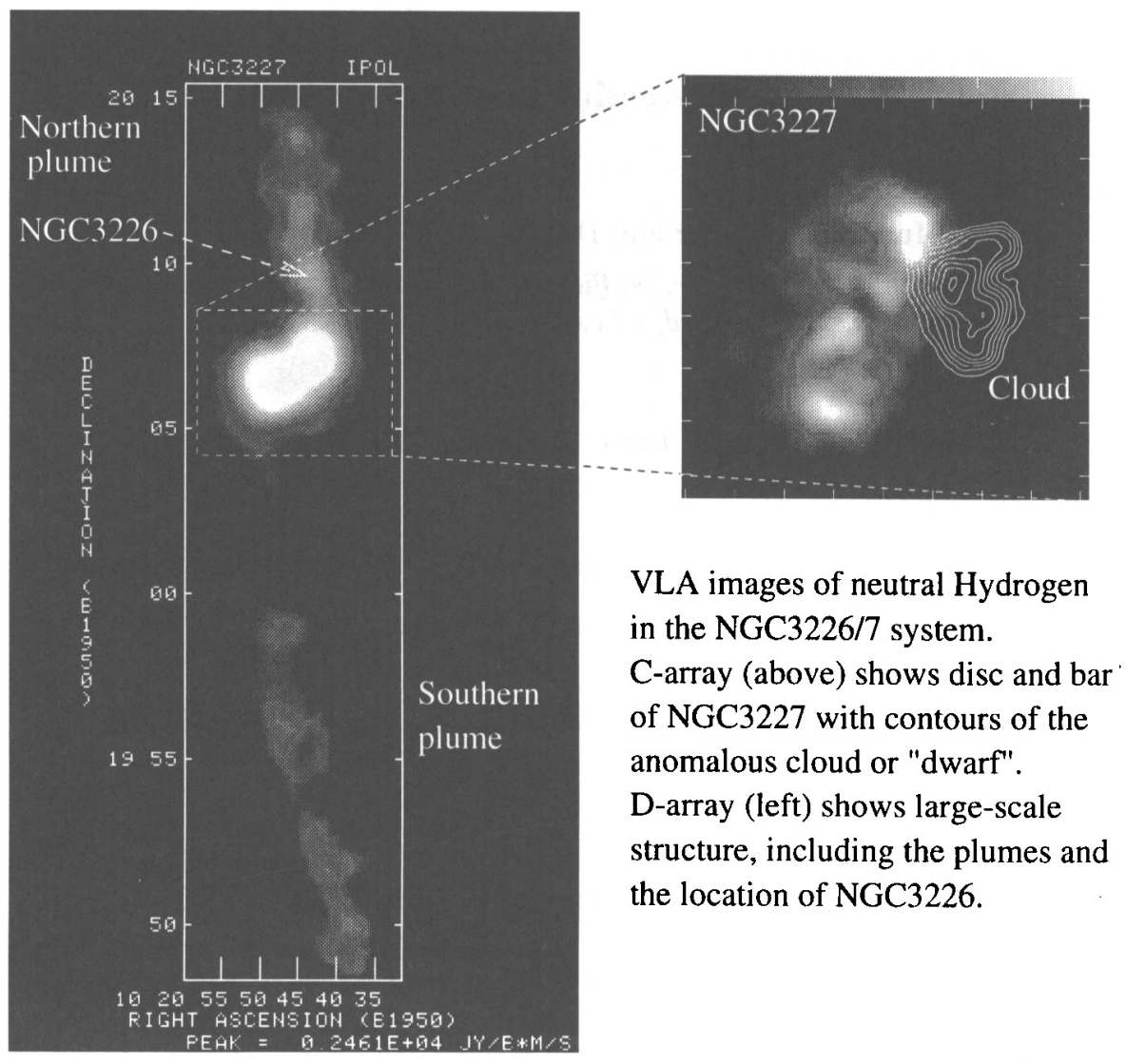

Figure 1. VLA Images of the NGC $3226 / 7$ system

partly responsible for the interaction or have been formed as a consequence of the interaction.

In NGC 3227, the HI bar crosses the nucleus from north-west to south-east and has a Z-shaped morphology which trails in the same sense as the optical spiral arms. This bar seems to be a continuation of a $\mathrm{CO}$ bar reported by Meixner et al. (1990).

Sundin et al. (1993) have shown that when a uniform disk is perturbed by a companion, a spiral pattern forms initially, which with time, starts to disperse as a bar begins to form in the center. Clearly, the interaction could have played a major role in the formation of the bar in NGC 3227.

Bars have been mooted for the transportation of material from large radii towards the nucleus of a galaxy (Schwarz 1985; Combes \& Gerin 1985; Athanassoula 1992; Barnes \& Hernquist 1992; Shaw et al. 1993), with the possibility of inflowing gas (on kiloparsec scales) accumulating in circumnuclear rings, barlike features or "spiral arms" (Athanassoula 1992; Vila-Viláro et al. 1994). It 
is unclear how the gas is driven further into the center, to subparsec scales, to fuel the active nucleus, but Shlosman et al. (1989) have suggested small bars on the nuclear scale, i.e., "bars within bars." Forbes et al. (1994) suggest that the presence of molecular bars may be a necessary (although perhaps not sufficient) requirement for nuclear activity.

In the present observations, the absence of any high-velocity gas, significant tidal disruption of the disk, or tidally-stripped gas being deposited directly onto the nucleus, leads us to the conclusion that the presence of the bar is crucial to the fuelling of the AGN.

\section{References}

Athanassoula, E. 1992, MNRAS, 259, 345

Barnes, J. E. \& Hernquist, L. 1992, Nature, 360, 715

Combes, F. \& Gerin, M. 1985, A\&A, 150, 327

Forbes, D. A., Kotilainen, J. K., \& Moorwood, A. F. M. 1994, ApJ, 433, L13

Meixner, M., Puchalsky, R., Blitz, L., Wright, M., \& Heckman, T. 1990, ApJ, 354,158

Mundell, C. G., Holloway, A. J., Pedlar, A., Meaburn, J., Kukula, M. J, \& Axon, D. J. 1995, MNRAS, 275, 67

Schwarz, M. P. 1985, MNRAS, 187, 73p

Shaw, M. A., Combes, F., Axon, D. J., \& Wright, G. S. 1993, A\&A, 273, 31

Shlosman, I., Frank, J., \& Begelman, M. C. 1989, Nature, 338, 45

Sundin, M., Donner, K. J., \& Sundelius, B. 1993, A\&A, 280, 105

Vila-Vilaró, B., Robinson, A., Perez, E., Axon, D. J., Baum, S. A., GonzálezDelgado, R. M., Pedlar, A. et al. 1995, A\&A, 302, 58V 